OPEN ACCESS

Edited by:

Andreas R. Luft,

University of Zurich, Switzerland

Reviewed by:

Tibor Auer,

University of Surrey, United Kingdom Alexandre Rosa Franco

Nathan Kline Institute for Psychiatric

Research, United States

*Correspondence:

Lejian Huang

lejian-huang@northwestern.edu

Songhe Jiang

jiangsonghe@wmu.edu.cn

tThese authors have contributed equally to this work and share last

authorship

Specialty section:

This article was submitted to

Stroke,

a section of the journa

Frontiers in Neurology

Received: 22 September 2021 Accepted: 17 January 2022

Published: 03 March 2022

Citation: Huang $Q$, Lin D, Huang S, Cao $Y$, Jin Y, Wu B, Fan L, Tu W, Huang L and Jiang S (2022) Brain Functional Topology Alteration in Right Lateral Occipital Cortex Is Associated With

Upper Extremity Motor Recovery.

Front. Neurol. 13:780966.

doi: 10.3389/fneur.2022.780966

\section{Brain Functional Topology Alteration in Right Lateral Occipital Cortex Is Associated With Upper Extremity Motor Recovery}

\author{
Qianqian Huang ${ }^{1,2}$, Dinghong Lin ${ }^{1,2}$, Shishi Huang ${ }^{3}$, Yungang $\mathrm{Cao}^{3}$, Yun $\mathrm{Jin}^{1,2}$, Bo $\mathrm{Wu}^{4}$, \\ Linyu Fan ${ }^{5}$, Wenzhan Tu ${ }^{1,2}$, Lejian Huang ${ }^{6 * t}$ and Songhe Jiang ${ }^{1,2 * t}$
}

${ }^{1}$ Rehabilitation Medicine Center, The Second Affiliated Hospital of Wenzhou Medical University, Wenzhou, China, ${ }^{2}$ Intelligent Rehabilitation Research Center, China-USA Institute for Acupuncture and Rehabilitation, Wenzhou Medical University, Wenzhou, China, ${ }^{3}$ Department of Neurology, The Second Affiliated Hospital and Yuying Children's Hospital of Wenzhou Medical University, Wenzhou, China, ${ }^{4}$ Department of Information, The Second Affiliated Hospital and Yuying Children's Hospital of Wenzhou Medical University, Wenzhou, China, ${ }^{5}$ Department of Radiology, The Second Affiliated Hospital and Yuying Children's Hospital of Wenzhou Medical University, Wenzhou, China, ${ }^{6}$ Department of Neuroscience, Feinberg School of Medicine, Northwestern University, Chicago, IL, United States

Stroke is a chief cause of sudden brain damage that severely disrupts the whole-brain network. However, the potential mechanisms of motor recovery after stroke are uncertain and the prognosis of poststroke upper extremity recovery is still a challenge. This study investigated the global and local topological properties of the brain functional connectome in patients with subacute ischemic stroke and their associations with the clinical measurements. A total of 57 patients, consisting of 29 left-sided and 28 right-sided stroke patients, and 32 age- and gender-matched healthy controls (HCs) were recruited to undergo a resting-state functional magnetic resonance imaging (rs-fMRI) study; patients were also clinically evaluated with the Upper Extremity Fugl-Meyer Assessment (FMA_UE). The assessment was repeated at 15 weeks to assess upper extremity functional recovery for the patient remaining in the study (12 left- 20 right-sided stroke patients). Global graph topological disruption indices of stroke patients were significantly decreased compared with HCs but these indices were not significantly associated with FMA_UE. In addition, local brain network structure of stroke patients was altered, and the altered regions were dependent on the stroke site. Significant associations between local degree and motor performance and its recovery were observed in the right lateral occipital cortex (R LOC) in the right-sided stroke patients. Our findings suggested that brain functional topologies alterations in R LOC are promising as prognostic biomarkers for right-sided subacute stroke. This cortical area might be a potential target to be further validated for non-invasive brain stimulation treatment to improve poststroke upper extremity recovery.

Keywords: stroke, upper extremity recovery, resting-state fMRI, right lateral occipital cortex, functional connectivity network 


\section{INTRODUCTION}

Stroke, a common medical emergency, remains the main cause of long-term disability and death in the world (1). Usually, stroke patients usually show hemiplegia, especially affecting the upper limb (2). Independence of the patients in activities of daily living depends on the recovery of their upper limb motor functions (3), and accurate prognosis of the recovery would enable realistic goal-setting and guide the allocation of rehabilitation resources. However, the prognosis of poststroke upper extremity recovery is still a challenge.

The most common neuroimaging biomarker to predict poststroke upper extremity recovery is measuring the integrity of the corticospinal tract (4-8). But neurological impairment poststroke can sometimes exceed expectations of stroke severity because stroke not only leads to focal, location-dependent neurological symptoms, but also causes widespread effects in remote hemispheres through functional networks (9-11). Researchers have recently discovered that along with the disorders of structural connectivity (12), reorganization of functional networks is related to the prognosis of patients (13-17). However, at present, there have been few reports on how the reorganization of brain functional networks predicts recovery of clinical motor function (18).

The properties of brain functional networks can be explored using graph theory. Resting-state fMRI (rs-fMRI) combined with network analysis demonstrated that the motor execution networks of convalescent stroke patients shifted to a more random topology (19); the local efficiency and small worldness of stroke patients were significantly higher and lower than those of healthy controls (HCs), respectively, and the latter was increased to a level close to that of the HCs during rehabilitation $(20,21)$. Moreover, an earlier study has shown functional network topological properties associated with poststroke outcomes in acute ischemic stroke patients (22). Therefore, we hypothesized that the brain functional topological properties were associated with an upper extremity motor recovery after stroke.

In this study, by taking advantage of a modest sample size (57 stroke patients and 32 age- and gender-matched HCs), global and local voxel-based graph theory analyses were performed and the correlation between brain network dysfunction and behavior in subacute stroke was fully investigated. First, after the patients were divided into two different subgroups based on stroke site, global graph properties of the two subgroups, and their association with clinical measurement were assessed independently. Following this, voxel-based degree maps between patients and their matched HCs were compared and association with clinical measurement was evaluated. Finally, using the significantly associated regions from the last step as regions of interest (ROIs), an ROI-based functional connectivity (FC) analysis was performed to explore the significantly different FC regions between patients and the HCs and found an ROI that can evaluate the extent to which upper extremity recovered postrehabilitation.

\section{MATERIALS AND METHODS}

\section{Participants}

We recruited 62 patients with first-time ischemic stroke between May 2017 and July 2020 at the Department of Physical Medicine and Rehabilitation in the Second Affiliated Hospital of Wenzhou Medical University, China. A total of five patients were removed from further data analyses because they did not pass our data quality control (see Section Quality control for details). To be eligible, participants must have (1) been between 30 and 85 years of age; (2) had their first stroke within the past month; (3) subcortical lesion restricted to the basal ganglia, internal capsule, corona radiate, or brainstem. Participants were excluded if they had the previous history of brain neurosurgery or epilepsy, or any MRI contraindications. The difference in lesion location of ischemic stroke is associated with different functional outcomes (23). Therefore, in this study, the stroke patients were divided into two subgroups: left-sided stroke patients and right-sided stroke patients.

In addition, 32 gender-, age-matched HCs were recruited. HCs were excluded if they (1) were $<30$ or more than 85 years old; (2) had psychiatric disease; (3) reported history of brain neurosurgical procedures and/or epilepsy; (4) were not suitable for MRI scan. All the stroke patients and HCs were right-handed. The demographical and clinical characteristics of stroke patients and HCs are detailed in Table $\mathbf{1}$.

This study was approved by the Institutional Review Board of the Second Affiliated Hospital and Yuying Children's Hospital of Wenzhou Medical University, China (Approval number: Clinical Scientific Research Ethical Review No. 2017LCKY-09) and all the participants provided written informed consent. All the research procedures were conducted in accordance with the Declaration of Helsinki.

\section{Clinical Assessment and Rehabilitation}

Clinical measurement related to motion performance was assessed. FMA_UE was used to measure upper limb movement, a highly recommended clinical tool for evaluating changes in motor impairment after stroke (range: 0-66) $(24,25)$. The upper extremity motor domain includes movement, coordination, and reflex action of the shoulder, elbow, forearm, wrist, and hand (24) and each item has a 3-point ordinal scale (0-cannot perform, 1performs partially, and 2-performs fully) (25). All the patients received two assessments of FMA_UE at the following time points: immediately following enrollment (baseline FMA_UE) and a follow-up 15 weeks after enrollment (follow-up FMA_UE). To evaluate the extent to which upper extremity was recovered postrehabilitation, FMA_UE recovery ratio was defined as

$$
\text { rFMA_UE }=\frac{\text { follow }- \text { up FMA_UE }- \text { baseline FMA_UE }}{\text { baseline FMA_UE }}
$$

and referred to as rFMA_UE. The positive and negative signs of rFMA_UE indicated improvement and deterioration of the upper extremity, respectively.

The FMA_UE assessments were evaluated by the same physician and collected on an electronic tablet device using Research Electronic Data Capture (REDCap) (26), a secure, 
TABLE 1 | Demographic and clinical characteristics of left-sided and right-sided stroke patients and HCs.

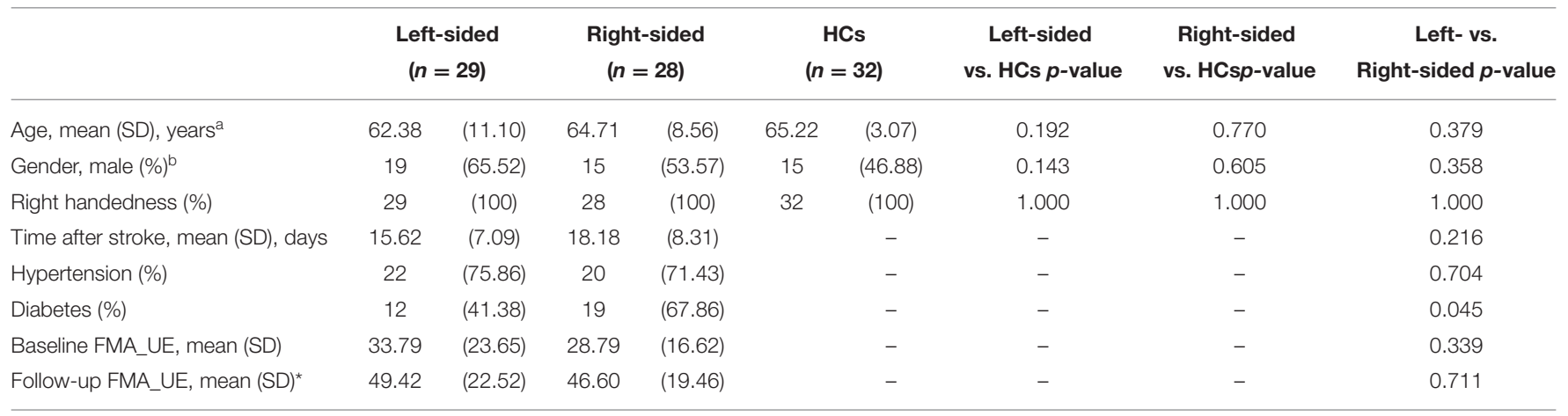

alndependent samples t-test.

${ }^{b}$ Chi-squared analysis.

SD, standard deviation; FMA_UE, Upper Extremity Fugl-Meyer Assessment.

- Unavailable; *Follow-up assessment was performed for 12 left-sided and 20 right-sided stroke patients.

convenient, and efficient web application for capturing electronic survey data.

All the patients received standard rehabilitation treatments after enrollment. The treatment program consists of physical and occupational therapy, including grips and finger movements, gross movement, stretching, and training in daily life activities.

\section{Magnetic Resonance Imaging Data Acquisition}

Subjects were scanned on a 3 Tesla GE-Discovery 750 scanner with the following parameters: for anatomical T1-MRI data: $\mathrm{TR} / \mathrm{TE}=7.7 / 3.4 \mathrm{~ms}$, flip angle $=12^{\circ}, \mathrm{FOV}=256 \times 256 \mathrm{~mm}$, resolution $=256 \times 256$, slice per volume $=176$, slice thickness $=$ $1 \mathrm{~mm}$; for $\mathrm{fMRI}$ data with odd interleave slice acquisition scheme: $\mathrm{TE} / \mathrm{TR}=30 / 2,500 \mathrm{~ms}$, voxel size $=3.4375 \times 3.4375 \times 3.5 \mathrm{~mm}^{3}$, in-plane resolution $=64 \times 64$, number of volumes $=230$, and flip angle $=90^{\circ}$.

\section{FMRI Data Preprocessing and Registration}

A scrubbing-based preprocessing method (27) was applied to all rs-fMRI data with the following steps: discard first four volumes; motion correction; slice-time correction; intensity normalization; high-pass temporal filtering $(0.008 \mathrm{~Hz})$ for correcting low-frequency signal drift; regression of six motion vectors, cerebrospinal fluid (CSF) signal-averaged overall voxels of eroded ventricle region, averaged white matter (WM) signal, and averaged global signal of whole brain; motion-volume censoring by detecting volumes with frame-wise displacement (FD) larger than $0.5 \mathrm{~mm}$, derivative variance root mean square (DVARS) after $Z$ normalization larger than 2.3, and SD after $Z$ normalization larger than 2.3 , and scrubbing above detected (number of volume $=i$ ) and adjacent four volumes $(i-2, i-1, i$, $i+1, i+2)(27,28)$. FD is a measure of head motion from one volume to the next, and is calculated as the sum of absolute value of three translational displacements in $x, y, z$ axis and three rotational displacements in pitch, yaw, and roll (units of radians), which were multiplied 50 to convert to similar units to translational displacements (27). DVARS is a measure of the change in volume intensity within a predefined gray matter (GM) mask from one volume to the next, calculated as the root mean square of the backward differentiated volumes; SD is a measure of deviation of volume intensity within the predefined GM mask. Because we were interested in the low-frequency fluctuations of resting-state fMRI signal, the aforementioned scrubbed time series were band-pass filtered $(0.008-0.1 \mathrm{~Hz})$ by applying a 4 thorder Butterworth filter.

All the preprocessed fMRI data were registered to MNI152 $2 \mathrm{~mm}$ template using a two-step procedure [ref. https://www. fmrib.ox.ac.uk/datasets/techrep/tr07ja2/tr07ja2.pdf]: the mean of preprocessed fMRI data were registered with a seven degrees of freedom affine transformation to its corresponding anatomical brain (FLIRT). Transformation parameters were also computed by non-linearly registering the individual brain to the MNI152 template (FNIRT). Combining the two transformations by matrix multiplication yielded transformation parameters normalizing fMRI data to standard space. All the final registered images were manually examined. Because the network analysis was voxel-wise-based, all registered images were down-sampled with linear interpolation to $6 \times 6 \times 6 \mathrm{~mm}^{3}$ for decreasing computational intensity.

We used 157 healthy subjects to generate an in-house $6 \times 6 \times$ 6 gray matter template. The procedure was briefly introduced, for each subject, first skull-stripped T1-brain image was segmented into gray matter (GM), white matter (WM), and cerebrospinal fluid (CSF) (FSL/FAST); second, GM was nonlinearly registered to MNI152_222 template (FSL/FNIRT); third, the 157 registered GMs were merged and averaged across subjects; the averaged GM was threshholded by $50 \%$ probability (the voxels were excluded if less than 79 subjects were covered). Final, the thresholded GM was downsampled to $6 \times 6 \times 6$.

\section{Quality Control}

After preprocessing, fMRI data were assessed for excessive motion. The number of censored motion volumes after preprocessing reflects the extent of motion of a subject during scanning. Any subject with $<120$ remaining volumes was excluded, which guaranteed a minimum of 5-min-scanning images for FC analysis (29). 


\section{Brain Functional Connectivity Network and Global Graph Properties}

Brain FC network that covered whole gray matter was constructed before global graph properties were computed. First, blood oxygenation level-dependent (BOLD) signal was extracted from each gray matter voxel in registered $6 \times$ $6 \times 6 \mathrm{~m}^{3}$ rs-fMRI data. Following this, a correlation matrix was generated by calculating voxel-based pairwise Pearson correlation coefficients of BOLD signals. Fisher's $z$ transformation was applied to convert the Pearson correlation coefficient. To normalize the variation of strength of brain FC across individuals, a link density, the percentage of links with respect to the maximum number of possible links, was predetermined, which corresponds to a threshold $(30,31)$. In this study, 10 link densities (from 1 to 10\%) were applied. Consequently, an indirectly connected brain FC network was generated after the correlation matrix was binarized by the subject-dependent threshold.

For each predetermined link density and each subject, five voxel-level graph properties were, respectively, computed using the brain connectivity toolbox (BCT) (32): "degree"-a measure of network hubness, "clustering coefficient"-a measure of network segregation, "betweenness centrality"-a measure of within-network communication, "efficiency"-a measure of network integration, and "participation coefficient" - a measure of diversity within a network.

\section{Graph Topological Disruption Index $\left(K_{D}\right)$ Comparison and Association With FMA_UE} $\mathrm{K}_{\mathrm{D}}$ is used to measure the extent of brain functional reorganization, describing the topological changes of individual brain networks with respect to referential network topology (31), and indicating brain network property changes of a subject in some regions with the opposite trend in other regions $(30,33,34)$. In this study, using brain of HCs as a normative reference, five graph topological disruption indices were computed: degree (D), betweenness centrality (BC), clustering coefficient (CC), efficiency (E), and participation coefficient (PC), and referred as $\mathrm{K}_{\mathrm{D} \_\mathrm{D}}, \mathrm{K}_{\mathrm{D} \_\mathrm{BC}}, \mathrm{K}_{\mathrm{D} \_\mathrm{CC}}, \mathrm{K}_{\mathrm{D} \_\mathrm{E}}$, and $\mathrm{K}_{\mathrm{D} \_\mathrm{PC}}$, respectively. For each graph topological disruption index, a repeated measures analysis of covariance (ANCOVA) with age and gender as covariates of no interest across all link density was performed to determine if there exists a significant difference between stroke patients (left-sided and right-sided, independently) and HCs. In addition, a Spearman correlation with age and gender as covariates was applied to examine if there exists a significant association between graph topological disruption indices and FMA_UE.

\section{Local Degree Comparison and Association With FMA_UE}

Because the five graph topological disruption indices are significantly correlated (35) and degree is an important marker of network development and resilience (32), degree was used for exploring brain FC locally. Degree map was generated from the brain FC network by counting the number of functional links on the gray matter at each voxel. To decrease the effect of motion on local FC (27), the links within two adjacent voxels were excluded. To identify voxel-wise degree differences between patients (life-sided or right-sided) and HCs, the script of randomize in FSL is performed to generate 5,000 permutations of the data while controlling for age and gender as confounds; family-wise correction (corrected $p=0.05$ ) was applied with threshold-free cluster enhancement (TFCE) (36). In addition, for each significant cluster found earlier, we further analyzed the association between the average degree count extracted from the cluster and FMA_UE. Using the script of fslmeants in FSL, the mean degree count across patient groups within each significant cluster was extracted, and Spearman correlation analysis was applied to examine if there exists a significant association between degree count and FMA_UE.

\section{Functional Connectivity Between Right Lateral Occipital Cortex (R LOC) and Other Nodes in the Subgroup of Right-Sided Stroke Patients}

After R LOC was discovered as the only region in which there existed significant association between local degree count and FMA_UE in the right-sided stroke patient subgroup, to explore which regions $\mathrm{R}$ LOC connected to and if these regions were significantly different between right-sided stroke patients and their age- and gender-matched HCs, R LOC was used as a region of interest (ROI) to generate ROI-based degree maps for both right-sided stroke patients and HCs. To identify the difference of ROI-based degree map between right-sided stroke patients and HCs, the script of randomize in FSL is performed to generate 5,000 permutations of the data while controlling for age and gender as confounds; family-wise correction (corrected $p=0.05)$ was applied with threshold-free cluster enhancement (TFCE) (36).

\section{Software}

All statistical analyses were performed using MATLAB 2016a, SPSS 23 (IBM Corp. in Armonk, NY), and FSL (www.fmrib.ox.ac.uk/fsl). Brain schemas of ROI and FC network were visualized on a surface rendering of a human brain atlas with the BrainNet Viewer (http://www.nitrc.org/projects/ bnv/) (37).

\section{RESULTS}

\section{Comparisons of Demographics Between Stroke Patients and HCs and Clinical Characteristics of the Patients}

As shown in Table 1, all 57 stroke patients were diagnosed with ischemic stroke. Of the 57 patients, 29 stroke patients had a leftsided stroke and the other 28 had a right-sided stroke, there were no statistical differences in age $(p=0.192,0.770)$ or gender $(p=0.143,0.605)$ between patients and their corresponding HCs. A total of 17 left- and 8 right-sided stroke patients did not participate in the follow-up assessment of FMA_UE. 


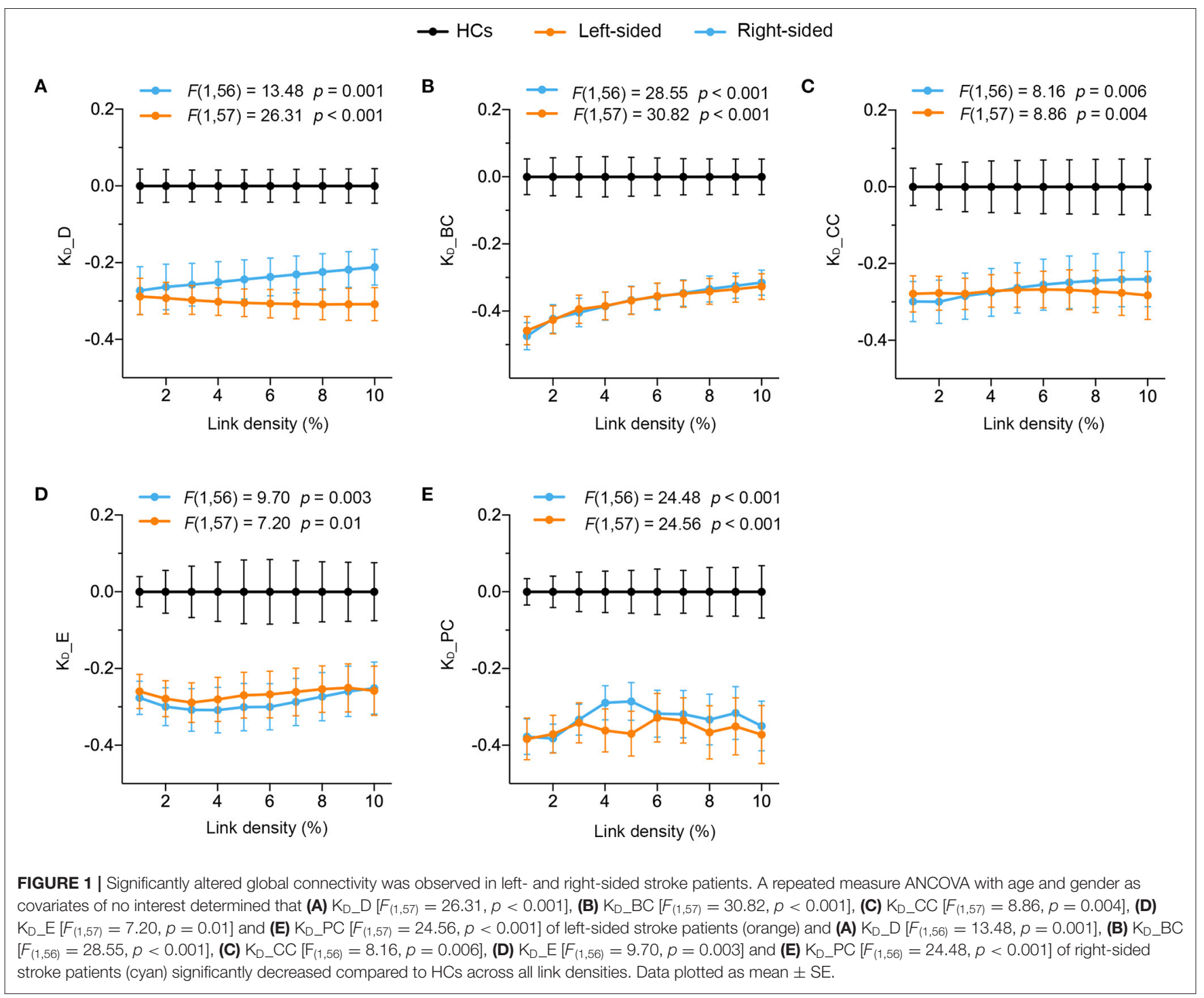

\section{Significantly Disrupted Global Brain Connectivity in Stroke Patients}

A repeated measures ANCOVA with age and gender as covariates of no interest determined that significant global altered connectivity was observed in stroke patients. All five graph topological disruption indexes of the left-sided stroke patients statistically significantly decreased compared to HCs across all link densities (from 1 to 10\%) (see Figure 1, orange). The same significant decrease was also observed in the right-sided stroke patients (see Figure 1, cyan). Therefore, we may conclude that global brain connectivity in both right- and left-sided stroke patients was significantly disrupted compared with that in HCs. However, across all the link densities, these five disruption indexes were not significantly associated with baseline FMA_UE for both right- and left-sided stroke patients (see Table 2), indicating that none of the global disruption indexes are promising predictors of motor impairment after stroke.
TABLE 2 | No significant correlations between the 5 disruption indexes in 2 subgroups and baseline FMA_UE at link density $=10 \%$.

\begin{tabular}{lccccc}
\hline & $\mathbf{K}_{\mathrm{D} \_} \mathbf{D}$ & $\mathbf{K}_{\mathrm{D} \_} \mathbf{B C}$ & $\mathbf{K}_{\mathrm{D} \_} \mathbf{C C}$ & $\mathbf{K}_{\mathrm{D} \_} \mathbf{E}$ & $\mathbf{K}_{\mathrm{D} \_} \mathbf{P C}$ \\
\hline $\begin{array}{l}\text { Left_sided } \\
R\end{array}$ & & & & & \\
$\begin{array}{l}P \text {-value } \\
\text { Right_sided }\end{array}$ & 0.651 & 0.835 & 0.876 & 0.661 & 0.254 \\
$R$ & & & & & \\
$P$-value & 0.202 & -0.035 & 0.111 & 0.130 & -0.056 \\
& 0.321 & 0.867 & 0.588 & 0.525 & 0.785 \\
\hline
\end{tabular}

$K_{D \_} D$, disruption index of degree; $K_{D \_} B C$, of betweenness centrality; $K_{D \_} C C$, of clustering coefficient; $K_{D} E$, of efficiency; $K_{D} P C$, of participation coefficient.

\section{Significantly Altered Local Connectivity in Stroke Patients}

In addition to the global connectivity alteration observed in stroke patients, we also found altered local connectivity measured by degree in stroke patients. Compared with HCs, patients with 
TABLE 3 | Brain regions with significant local degree differences between stroke patients and HCs.

\begin{tabular}{|c|c|c|c|c|c|c|}
\hline \multirow[t]{2}{*}{ Brain regions } & \multicolumn{3}{|c|}{ MNI coordinates } & \multirow[t]{2}{*}{ Cluster size $\left(\mathrm{mm}^{3}\right)$} & \multirow[t]{2}{*}{$t_{(59)}$-value } & \multirow[t]{2}{*}{$p$-value } \\
\hline & $\mathbf{x}$ & $\mathbf{Y}$ & $\mathbf{z}$ & & & \\
\hline \multicolumn{7}{|c|}{ (A) Brain regions with significant local degree differences between left-sided patients and HCs at $10 \%$ link density } \\
\hline \multicolumn{7}{|l|}{ Stroke Patients > HCs } \\
\hline Left precentral gyrus & -42 & -6 & 36 & 1,296 & 4.12 & $<0.001$ \\
\hline Right amygdala & 30 & 0 & -24 & 864 & 4.17 & $<0.001$ \\
\hline \multicolumn{7}{|l|}{ Stroke Patients $<$ HCs } \\
\hline Left precuneous cortex & 0 & -60 & 42 & 9,504 & 4.25 & $<0.001$ \\
\hline Right lateral occipital cortex ${ }^{\dagger}$ & 18 & -84 & 42 & 3,240 & 4.77 & $<0.001$ \\
\hline Right supramarginal gyrus ${ }^{\ddagger}$ & 60 & -42 & 36 & 2,808 & 4.42 & $<0.001$ \\
\hline Left occipital pole ${ }^{\ddagger}$ & -18 & -90 & 36 & 1,944 & 4.29 & $<0.001$ \\
\hline Right lateral occipital cortex ${ }^{\dagger}$ & 60 & -66 & 0 & 1,080 & 3.90 & $<0.001$ \\
\hline Left lateral occipital cortex & -48 & -78 & 6 & 648 & 3.64 & $<0.001$ \\
\hline
\end{tabular}

$t_{(59)}$ : 59 represents degree of freedom. ${ }^{*}$ : overlapped region with that from right-sided patients

(B) Brain regions with significant local degree differences between right-sided patients and HCs at $10 \%$ link density Stroke Patients $>\mathrm{HCs}$

Left precentral gyrus

Right inferior frontal gyrus

Left superior temporal gyrus

Left inferior frontal gyrus

Stroke Patients $<$ HCs

Right occipital pole ${ }^{\ddagger}$

Right supramarginal gyrus ${ }^{\ddagger}$

Right lateral occipital cortex ${ }^{\dagger}$

Left lateral occipital cortex $^{*}$

$\begin{array}{ccc}-12 & -30 & 48 \\ 48 & 18 & 18 \\ -60 & -18 \\ -42 & 18 \\ & & 12 \\ 24 & -90 & 3 \\ 66 & -30 & 30 \\ 60 & -66 & \\ -18 & -84 & 24\end{array}$

$\begin{array}{cc}48 & 1,512 \\ 18 & 864 \\ 0 & 648 \\ 12 & 648 \\ & \\ 36 & 6,264 \\ 30 & 3,024 \\ 6 & 2,592 \\ 24 & 864\end{array}$

4.77

$<0.001$

0.002

$<0.001$

$<0.001$

$t_{(58)}, 58$ represents degree of freedom; ${ }^{\ddagger}$ Overlapped region with that from left-sided patients. Bold values of $p$-value represents statistically significance $(p<0.05)$.

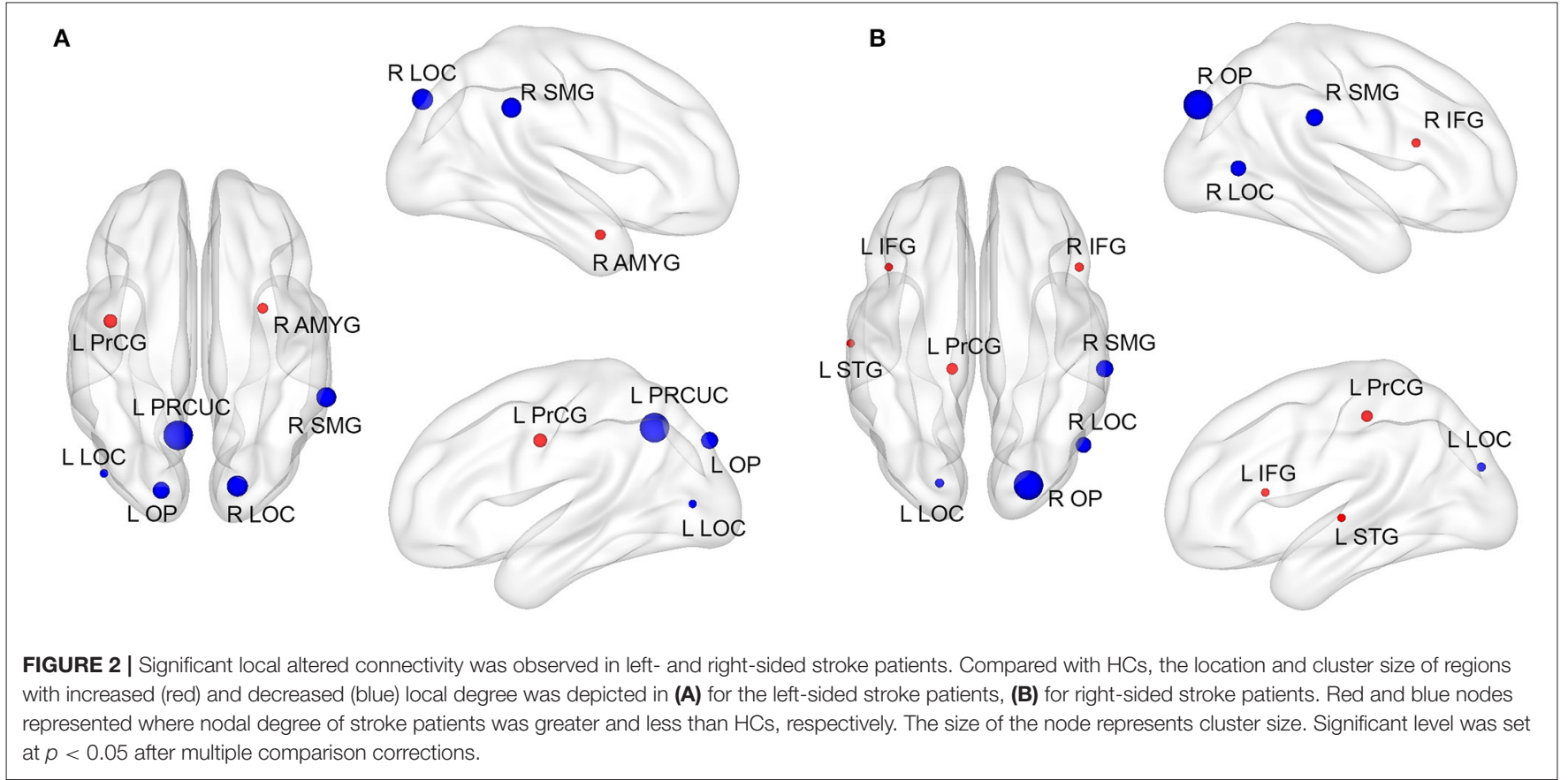

left-sided stroke exhibited significantly increased nodal degree in the left precentral gyrus (L PrCG) and right amygdala (R AMYG), and the decreased nodal degree in the left precuneous cortex (L PRCUC), right supramarginal gyrus (R SMG), left occipital pole (LOP), right lateral occipital cortex (R LOC), and L LOC (Table 3A; Figure 2A). Patients with right-side stroke 
showed significantly increased nodal degree in the L PrCG, right inferior frontal gyrus (R IFG), left superior temporal gyrus (L STG) and L IFG, and the decreased nodal degree in the right occipital pole (R OP), R SMG, R LOC, and L LOC (Table 3B; Figure 2B).

\section{Degree in R LOC Was Associated With Baseline FMA_UE and Predicted the Extent of Motion Recovery in the Right-Sided Stroke Patients}

We further analyzed the associations between the local degree count extracted from significant brain regions (Table 2B; Figure 3 ) in both left- and right-sided stroke patients and their baseline FMA_UEs. Only R LOC in the group of the rightsided stroke patient was significant after a Bonferroni correction $\left(p_{\text {Bonferroni }}=0.006\right)(\rho=-0.861, p<0.001$, Figure 3A). Moreover, the degree in R LOC was able to predict rFMA_UE, the extent of upper extremity recovery after rehabilitation ( $\rho=$ 0.691, $p=0.001$, Figure 3B).

\section{ROI-Based FC Analysis Revealed Regions With Significantly Different FC in Right-Sided Stroke Patients}

By using R LOC as an ROI, the region in which local degree alteration was associated with motor performance, an ROI-based FC analysis revealed regions with significantly different FC in right-sided stroke patients compared with HCs. The regions with increased degree connectivity to R LOC were L PRCUC, right middle frontal gyrus (R MFG), and left middle frontal gyrus ( $\mathrm{L}$ MFG). The regions with decreased degree connectivity to R LOC are the left supplementary motor cortex (L SMA), left postcentral gyrus (L PoCG), R SMG, R PrCG, and right superior frontal gyrus (R SFG) (Figure 4). The detailed MNI coordinates and the cluster size are listed in Table 4.

\section{DISCUSSION}

A key theoretical and clinical question of interest is the nature of any topological abnormalities in the brain network organization of stroke patients which might relate to their motor performance; assessing this would shed light on which aspects of normal brain network organization might be critical for upper limb motor recovery.

In this study, we applied graph-based theoretical approaches to rs-fMRI to analyze the brain functional connectome topology in subacute ischemic stroke compared with HCs. Our main findings are summarized as follows: (1) at the global level, using brain of $\mathrm{HC}$ as a normative reference, there exists significantly disrupted global brain connectivity in subacute stroke patients compared with HCs, but no correlation between global disruption indexes and clinical measurements was found; (2) at the local nodal level, by measuring degree, significant local degree differences between stroke patients and HCs were found, among which baseline FMA_UE and rFMA_UE were significantly correlated to the degree count in R LOC in rightsided stroke patients.
We found that whole-brain network structure of the stroke patients was altered, regardless of stroke site. Graph disruption indices derived from five network topological measurements, degree, clustering coefficient, participation coefficient, betweenness centrality and efficiency, which, respectively, represent network hubness, segregation, diversity, withinnetwork communication, and integration, were significantly decreased compared with HCs across all predefined link densities in two subgroups. The results, in accord with findings of Termenon et al. (33) of significantly lower $K_{D}$ in the contralesional hemispheres of brain networks of the patients, indicates that stroke-induced whole brain reorganization, suggesting that FC and other network properties vary in the certain regions at the subacute stage. The failure to observe any significant association between global hub disruption and FMA_UE across two subgroups reveals that although global hub disruption is a more reliable and sensitive metric than graph metrics to detect brain reorganization (33), it is not a biomarker to predict the motor dysfunction caused by stroke at the subacute stage, which implies that the clinical measurement reflecting motor dysfunction might only be related to brain reorganization within brain network(s) rather than whole brain reorganization.

We found that local-brain network structure of stroke patients was altered in terms of degree compared with HCs. From data shown in Figure 2, we conclude that the local altered regions were not identical across two subgroups. In general, the increased degree was mainly located in the temporal and frontal lobes, decreased in the occipital lobe, and altered regions were located not only in the hemisphere of the lesion, but also in the contralesional hemisphere. These findings might reflect the overall impact of local lesions on the long-distance functional connective area and indicate that the visual, motor, emotional, language processing, and cognitive processes of patients with subacute ischemic stroke were damaged or at least degraded.

The right-sided stroke patients, where the degree extracted from degree-altered regions was significantly correlated to the upper extremity motor function, showed significantly decreased degree in the occipital cortex and right supramarginal gurus, and increased degree mainly in the L PrCG, R IFG, L STG, and L IFG (see Figure 2B; Table 3B). The observation of decrement of degree in the occipital cortex is consistent with findings in patients with transient ischemic attack (TIA) (38) and ischemic stroke patients (39), as this region is a part of the visual dorsal stream and is involved in object localization (40). The right-sided stroke patients with more severe paralysis (lower FMA_UE) had more FC in the R-LOC (Figure 3A), which could be attributed to functional compensation after the onset of stroke (41). Similarly, for the regions with increased degree, previous studies have demonstrated that altered organization of connectivity in the middle frontal gyrus in patients with subcortical stroke reflected the role of the frontal lobe in higher-order movement planning $(42,43)$, as the frontal lobe is associated with the multiple forms of olfaction and higher-order cognition, including working memory, inhibitory control, conflict monitoring, and shifting between rule sets (44), and that the L PrCG is in the sensorimotor network (SMN), a network with multifunctional characteristics and vulnerable not only to neurodegenerative diseases, but also to 

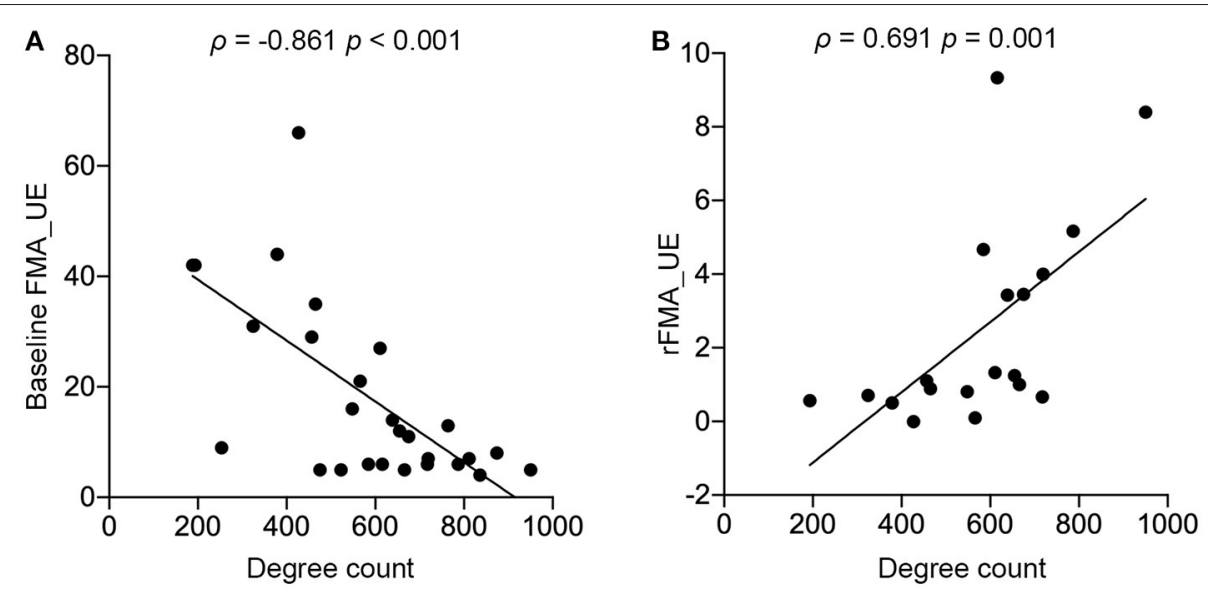

FIGURE 3 | Significant associations between degree and upper extremity motor performance were discovered in R LOC in the right-sided stroke patients. (A) Baseline FMA_UE scores significantly correlated to local degree in R LOC; (B) rFMA_UE significantly correlated to local degree in R LOC.

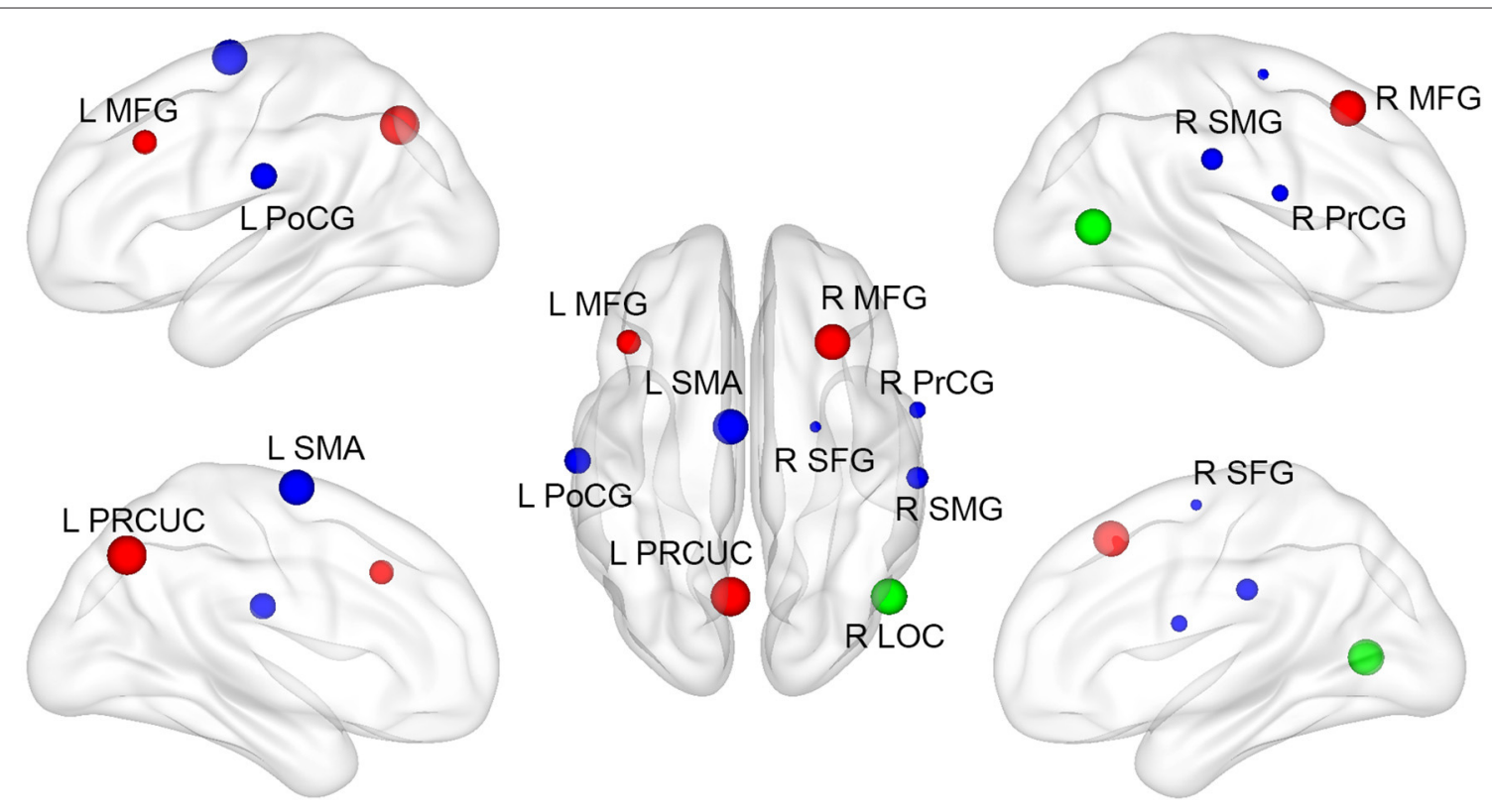

FIGURE 4 | Regions with significantly different ROI-based functional connectivity in right-sided stroke patients. Compared to HCs, the location and cluster size of regions with significantly different $\mathrm{R}$ LOC-based functional connectivity. Red and blue nodes represent those regions of which functional connections were greater and less than HCs, respectively. The green node represents the seed ROI (R LOC).

cerebrovascular diseases $(45,46)$. The compensatory mechanism for motor impairment might contribute to this degree increment. However, we did not observe the same associations in the left-sided stroke patients. One of the possible reasons might be that left- and right-side stroke patients have significantly different topological properties $(47,48)$. Left-lateralization in the language process (49) and the left hemisphere being the dominant hemisphere of right-handers might result in the leftside stroke patients having more complex FC changes poststroke.

Our finding that the degree extracted from the R LOC region was significantly associated with upper extremity motor recovery [i.e., right-sided stroke patients with more severe paralysis had more potential to benefit from the compensatory mechanism, leading to a higher chance of FC in R LOC after 15 weeks rehabilitation (Figure 3B)], assists our understanding of the importance of eyehand coordination in the rehabilitation of upper limb motor function. The R LOC is part of the visual cortex (40) that connects with the amygdala, hippocampus, and via intrathalamic pathways with mediofrontal areas (50). The R LOC also receives top-down modulation from frontal and parietal areas in relation to visual attention (51). Previous studies indicate visual feedback can enhance motor function 
TABLE 4 | Brain regions with significant differences in R LOC functional connectivity between right-sided stroke patients and HCs.

\begin{tabular}{|c|c|c|c|c|c|c|}
\hline & \multicolumn{3}{|c|}{ MNI coordinates } & \multirow[t]{2}{*}{ Cluster size $\left(\mathrm{mm}^{3}\right)$} & \multirow[t]{2}{*}{$t_{(58)}$-value } & \multirow[t]{2}{*}{$p$-value } \\
\hline & $\mathbf{x}$ & $\mathbf{Y}$ & $\mathbf{Z}$ & & & \\
\hline \multicolumn{7}{|l|}{ Stroke Patients > HCs } \\
\hline Left precuneus & -6 & -66 & 42 & 8,640 & 4.19 & $<0.001$ \\
\hline Right middle frontal gyrus & 30 & 24 & 48 & 6,264 & 5.64 & $<0.001$ \\
\hline Left middle frontal gyrus & -42 & 24 & 36 & 2,160 & 3.57 & $<0.001$ \\
\hline \multicolumn{7}{|l|}{ Stroke Patients < HCs } \\
\hline Left supplementary motor cortex & -6 & -6 & 66 & 6,048 & 3.53 & $<0.001$ \\
\hline Left postcentral gyrus & -60 & -18 & 24 & 2,592 & 4.51 & $<0.001$ \\
\hline Right supramarginal gyrus & 66 & -24 & 30 & 1,728 & 4.03 & $<0.001$ \\
\hline Right precentral gyrus & 60 & 0 & 18 & 1,080 & 4.07 & $<0.001$ \\
\hline Right superior frontal gyrus & 24 & -6 & 60 & 648 & 3.30 & 0.002 \\
\hline
\end{tabular}

Bold values of $p$-value represents statistically significance $(p<0.05)$.

$(52,53)$ so that during rehabilitation, dependence on visual feedback increases $(54,55)$ and viewing motion stimuli leads to activity increases in regions of the extrastriate visual cortex (56). Moreover, multimodal fMRI experiments in (57) revealed that passive touch significantly activated the object selective parts of LOC and that the coupling was specific to hand and shoulder stimulation, suggesting that LOC is functionally connected to the hand area of primary somatosensory. While our data cannot provide causality direction between R LOC and upper extremity motor recovery, thereby preventing us from identifying it as a sure brain region to target for treatment, together, these findings, and the previous finding point to the R LOC as an important region to further probe for intervention methods of poststroke motor rehabilitation.

By virtue of an R LOC-based FC analysis, we identified the regions that connected to $\mathrm{R}$ LOC and the degree extracted from which was significantly increased compared to HCs at the subacute stage poststroke, left PRCUC and left and right MFG, and the regions with decreased connectivity, L SMA, L PoCG, R SMG, R PrCG, and R SFG. These regions correlated with the motor recovery after stroke $(20,58,59)$. Combined with the discovery that the more the degree was extracted from the R LOC, the better motor function recovery the patient demonstrated, these widespread regions in both hemispheres might provide subacute stroke patients with a motor compensation mechanism after the onset of stroke.

There are limitations in this study. First, in our study, 74 and $54 \%$ of patients had hypertension, and diabetes, respectively, so variances in brain structure and function caused by hypertension (60), diabetes (61), and stroke are difficult to delineate. In the future, we will have a cross-sectional study on the differences between groups of stroke patients with and without hypertension and diabetes. Second, participants were not divided into groups according to the severity of motor dysfunction. Third, our sample size of 28 right-sided stroke patients is relatively small; future work will include a larger cohort of patients; and focus on the analysis of longitudinal changes in brain regions critical to stroke outcomes captured through brain functional topological properties. Fourth, we did not collect fMRI data during the follow-up period, and thus cannot detect how brain networks reorganize as stroke continues to evolve. Fifth, we did not collect language-related measurements, which prevented us from further investigating why left- and right-sided stroke patients had different associations between the degree extracted from R LOC and the upper extremity motor recovery.

\section{CONCLUSIONS}

In conclusion, the five global graph topological disruption indices of stroke patients were significantly decreased in two subgroups compared with HCs. Correlation analysis revealed that these global disruption indices were not significantly associated with FMA_UE. Local brain FC in terms of degree was explored and it was found that local brain network structure of the stroke patients was altered and significant associations between region degree and upper extremity motor functional recovery were observed in R LOC in the subgroup of right-sided stroke patients. These findings suggest that the topological properties of functional brain networks may provide prognostic value for motor functional recovery after stroke.

\section{DATA AVAILABILITY STATEMENT}

The original contributions presented in the study are included in the article/supplementary material, further inquiries can be directed to the corresponding authors.

\section{ETHICS STATEMENT}

The studies involving human participants were reviewed and approved by Institutional Review Board of the Second Affiliated Hospital and Yuying Children's Hospital of Wenzhou Medical University. The patients/participants provided their written informed consent to participate in this study. 


\section{AUTHOR CONTRIBUTIONS}

SJ, $\mathrm{LH}$, and $\mathrm{QH}$ contributed to conception and study design. WT, DL, YC, YJ, and LF contributed to acquisition of data. QH, LH, $\mathrm{SH}$, and $\mathrm{BW}$ analyzed functional images and statistical analysis. $\mathrm{LH}$ and $\mathrm{QH}$ drafted and edited the manuscript. All authors approved the final manuscript version to be published.

\section{FUNDING}

QH received a grant from the Wenzhou Bureau of Science and Technology (No. Y20190042). SJ received a

\section{REFERENCES}

1. Powers WJ, Rabinstein AA, Ackerson T, Adeoye OM, Bambakidis NC, Becker $\mathrm{K}$, et al. Guidelines for the early management of patients with acute ischemic stroke: 2019 update to the 2018 Guidelines for the early management of acute ischemic stroke: a guideline for healthcare professionals from the American Heart Association/American Stroke Association. Stroke. (2019) 50:e344-418. doi: 10.1161/STR.0000000000000211

2. Duncan PW, Goldstein LB, Matchar D, Divine GW, Feussner J. Measurement of motor recovery after stroke. Outcome assessment and sample size requirements. Stroke. (1992) 23:1084-9. doi: 10.1161/01.STR.23.8.1084

3. Veerbeek JM, Kwakkel G, van Wegen EE, Ket JC, Heymans MW. Early prediction of outcome of activities of daily living after stroke: a systematic review. Stroke. (2011) 42:1482-8. doi: 10.1161/STROKEAHA.110.60 4090

4. Hodgson K, Adluru G, Richards LG, Majersik JJ, Stoddard G, Adluru N, et al. Predicting motor outcomes in stroke patients using diffusion spectrum MRI microstructural measures. Front Neurol. (2019) 10:72. doi: 10.3389/fneur.2019.00072

5. Cassidy J, Tran G, Quinlan E, Cramer S. Neuroimaging identifies patients most likely to respond to a restorative stroke therapy. Stroke. (2018) 49:4338. doi: 10.1161/STROKEAHA.117.018844

6. Puig J, Blasco G, Schlaug G, Stinear C, Daunis-I-Estadella P, Biarnes C, et al. Diffusion tensor imaging as a prognostic biomarker for motor recovery and rehabilitation after stroke. Neuroradiology. (2017) 59:34351. doi: 10.1007/s00234-017-1816-0

7. Groisser B, Copen W, Singhal A, Hirai K, Schaechter J. Corticospinal tract diffusion abnormalities early after stroke predict motor outcome. Neurorehabil Neural Repair. (2014) 28:751-60. doi: 10.1177/1545968314521896

8. Rosso C, Lamy JC. Prediction of motor recovery after stroke: being pragmatic or innovative? Curr Opin Neurol. (2020) 33:482-7. doi: 10.1097/WCO.0000000000000843

9. Sun L, Yin D, Zhu Y, Fan M, Zang L, Wu Y, et al. Cortical reorganization after motor imagery training in chronic stroke patients with severe motor impairment: a longitudinal fMRI study. Neuroradiology. (2013) 55:91325. doi: 10.1007/s00234-013-1188-Z

10. Ward N, Brown M, Thompson A, Frackowiak R. Neural correlates of motor recovery after stroke: a longitudinal fMRI study. Brain. (2003) 126:247696. doi: 10.1093/brain/awg245

11. Grefkes C, Fink G. Connectivity-based approaches in stroke and recovery of function. Lancet Neurol. (2014) 13:20616. doi: 10.1016/S1474-4422(13)70264-3

12. Moulton E, Amor-Sahli M, Perlbarg V, Pires C, Crozier S, Galanaud D, et al. Axial diffusivity of the corona radiata at 24 hours post-stroke: a new biomarker for motor and global outcome. PLoS ONE. (2015) 10:e0142910. doi: 10.1371/journal.pone.0142910

13. Loubinoux I, Carel C, Pariente J, Dechaumont S, Albucher J, Marque $\mathrm{P}$, et al. Correlation between cerebral reorganization and motor recovery after subcortical infarcts. Neuroimage. (2003) 20:2166-80. doi: 10.1016/j.neuroimage.2003. 08.017 grant from the Medical Health Science and Technology Project of Zhejiang Provincial Health Commission (No. 2017198456).

\section{ACKNOWLEDGMENTS}

We would thank Le Lin, Xinxin Lin, and Huifang $\mathrm{Li}$ at the Department of Physical Medicine and Rehabilitation in the Second Affiliated Hospital of Wenzhou Medical University Management for helping with recruitment.
14. Jaillard A, Martin C, Garambois K, Lebas J, Hommel M. Vicarious function within the human primary motor cortex? A longitudinal fMRI stroke study. Brain. (2005) 128:1122-38. doi: 10.1093/brain/awh456

15. Gerloff C, Bushara K, Sailer A, Wassermann E, Chen R, Matsuoka T, et al. Multimodal imaging of brain reorganization in motor areas of the contralesional hemisphere of well recovered patients after capsular stroke. Brain. (2006) 129:791-808. doi: 10.1093/brain/awh713

16. Favre I, Zeffiro T, Detante O, Krainik A, Hommel M, Jaillard A. Upper limb recovery after stroke is associated with ipsilesional primary motor cortical activity: a meta-analysis. Stroke. (2014) 45:1077-83. doi: 10.1161/STROKEAHA.113.003168

17. Shan Y, Wang YS, Zhang M, Rong DD, Zhao ZL, Cao YX, et al. Homotopic connectivity in early pontine infarction predicts late motor recovery. Front Neurol. (2018) 9:907. doi: 10.3389/fneur.2018.00907

18. Boyd L, Hayward K, Ward N, Stinear C, Rosso C, Fisher R, et al. Biomarkers of stroke recovery: consensus-based core recommendations from the Stroke Recovery and Rehabilitation Roundtable. Int J Stroke. (2017) 12:480-93. doi: 10.1177/1747493017714176

19. Wang L, Yu C, Chen H, Qin W, He Y, Fan F, et al. Dynamic functional reorganization of the motor execution network after stroke. Brain. (2010) 133(Pt 4):1224-38. doi: 10.1093/brain/awq043

20. Yin D, Song F, Xu D, Sun L, Men W, Zang L, et al. Altered topological properties of the cortical motor-related network in patients with subcortical str oke revealed by graph theoretical analysis. Hum Brain Mapp. (2014) 35:3343-59. doi: 10.1002/hbm.22406

21. Siegel JS, Seitzman BA, Ramsey LE, Ortega M, Gordon EM, Dosenbach NUF, et al. Re-emergence of modular brain networks in stroke recovery. Cortex. (2018) 101:44-59. doi: 10.1016/j.cortex.2017.12.019

22. Ktena SI, Schirmer MD, Etherton MR, Giese AK, Tuozzo C, Mills BB, et al. Brain connectivity measures improve modeling of functional outcome after acute ischemic stroke. Stroke. (2019) 50:2761-7. doi: 10.1161/STROKEAHA.119.025738

23. Cheng B, Forkert N, Zavaglia M, Hilgetag C, Golsari A, Siemonsen $\mathrm{S}$, et al. Influence of stroke infarct location on functional outcome measured by the modified rankin scale. Stroke. (2014) 45:1695-702. doi: 10.1161/STROKEAHA.114.005152

24. Gladstone DJ, Danells CJ, Black SE. The fugl-meyer assessment of motor recovery after stroke: a critical review of its measurement proper ties. Neurorehabil Neural Repair. (2002) 16:232-40. doi: 10.1177/154596802401105171

25. Fugl-Meyer AR, Jääskö L, Leyman I, Olsson S, Steglind S. The post-stroke hemiplegic patient. 1. A method for evaluation of physical performance. Scand J Rehabil Med. (1975) 7:13-31.

26. Harris P, Taylor R, Thielke R, Payne J, Gonzalez N, Conde J. Research electronic data capture (REDCap)-a metadata-driven methodology and workflow process for providing translational research informatics support. $J$ Biomed Inform. (2009) 42:377-81. doi: 10.1016/j.jbi.2008.08.010

27. Power JD, Mitra A, Laumann TO, Snyder AZ, Schlaggar BL, Petersen SE. Methods to detect, characterize, and remove motion artifact in resting state fMRI. Neuroimage. (2014) 84:320-41. doi: 10.1016/j.neuroimage.2013.08.048

28. Power JD, Barnes KA, Snyder AZ, Schlaggar BL, Petersen SE Spurious but systematic correlations in functional connectivity 
MRI networks arise from subject motion. Neuroimage. 59:2142-54. doi: 10.1016/j.neuroimage.2011.10.018

29. Yang L, Wu B, Fan L, Huang S, Vigotsky A, Baliki M, et al. Dissimilarity of functional connectivity uncovers the influence of participant's motion in functional magnetic resonance imaging studies. Hum Brain Mapp. (2020) 42:713-23. doi: 10.1002/hbm.25255

30. Mansour A, Baria A, Tetreault P, Vachon-Presseau E, Chang P, Huang L, et al. Global disruption of degree rank order: a hallmark of chronic pain. Sci Rep. (2016) 6:34853. doi: 10.1038/srep34853

31. Achard S, Delon-Martin C, Vertes PE, Renard F, Schenck M, Schneider F, et al. Hubs of brain functional networks are radically reorganized in comatose patients. Proc Natl Acad Sci USA. (2012) 109:2060813. doi: 10.1073/pnas.1208933109

32. Rubinov M, Sporns O. Complex network measures of brain connectivity: uses and interpretations. Neuroimage (2010) 52:1059-69. doi: 10.1016/j.neuroimage.2009.10.003

33. Termenon M, Achard S, Jaillard A, Delon-Martin C. The "Hub Disruption Index," a reliable index sensitive to the brain networks reorganization. A study of the contralesional hemisphere in stroke. Front Comput Neurosci. (2016) 10:84. doi: 10.3389/fncom.2016.00084

34. Mano H, Kotecha G, Leibnitz K, Matsubara T, Sprenger C, Nakae A, et al. Classification and characterisation of brain network changes in chronic back pain: a multicenter study. Wellcome Open Res. (2018) 3:19. doi: 10.12688/wellcomeopenres.14069.2

35. Huang S, Wakaizumi K, Wu B, Shen B, Wu B, Fan L, et al. Whole-brain functional network disruption in chronic pain with disk herniation. Pain. (2019) 160:2829-40. doi: 10.1097/j.pain.0000000000001674

36. Smith SM, Nichols TE. Threshold-free cluster enhancement: addressing problems of smoothing, threshold dependence and locali sation in cluster inference. Neuroimage. (2009) 44:8398. doi: 10.1016/j.neuroimage.2008.03.061

37. Xia M, Wang J, He Y. BrainNet Viewer: a network visualization tool for human brain connectomics. PLoS ONE. (2013) 8:e68910. doi: 10.1371/journal.pone.0068910

38. Lv Y, Han X, Song Y, Han Y, Zhou C, Zhou D, et al. Toward neuroimagingbased network biomarkers for transient ischemic attack. Hum Brain Mapp. (2019) 40:3347-61. doi: 10.1002/hbm.24602

39. Zhang J, Zhang Y, Wang L, Sang L, Yang J, Yan R, et al. Disrupted structural and functional connectivity networks in ischemic stroke patients. Neuroscience. (2017) 364:212-25. doi: 10.1016/j.neuroscience.2017.09.009

40. Wandell BA, Dumoulin SO, Brewer AA. Visual field maps in human cortex. Neuron. (2007) 56:366-83. doi: 10.1016/j.neuron.2007.10.012

41. Zhu Y, Bai L, Liang P, Kang S, Gao H, Yang H. Disrupted brain connectivity networks in acute ischemic stroke patients. Brain Imaging Behav. (2017) 11:444-53. doi: 10.1007/s11682-016-9525-6

42. Park $\mathrm{CH}$, Chang WH, Ohn SH, Kim ST, Bang OY, PascualLeone A, et al. Longitudinal changes of resting-state functional connectivity during motor recovery after stroke. Stroke. (2011) 42:1357-62. doi: 10.1161/STROKEAHA.110.596155

43. Yin D, Song F, Xu D, Peterson B, Sun L, Men W, et al. Patterns in cortical connectivity for determining outcomes in hand function after subcortical stroke. PLoS ONE. (2012) 7:e52727. doi: 10.1371/journal.pone.0052727

44. Miyake A, Friedman NP. The nature and organization of individual differences in executive functions: four general conclusions. Curr Dir Psychol Sci. (2012) 21:8-14. doi: 10.1177/0963721411429458

45. Dipasquale O, Griffanti L, Clerici M, Nemni R, Baselli G, Baglio F. Highdimensional ICA analysis detects within-network functional connectivity damage of default-mode a nd sensory-motor networks in Alzheimer's disease. Front Hum Neurosci. (2015) 9:43. doi: 10.3389/fnhum.2015.00043

46. Wu X, Lai Y, Zhang Y, Yao L, Wen X. Breakdown of sensorimotor network communication in leukoaraiosis. Neurodegener Dis. (2015) 15:32230. doi: 10.1159/000435918

47. Shi M, Liu S, Chen H, Geng W, Yin X, Chen YC, et al. Disrupted brain functional network topology in unilateral acute brainstem ischemic stroke. Brain Imaging Behav. (2020) 15:444-52. doi: 10.1007/s11682-02000353-z

48. Caliandro P, Vecchio F, Miraglia F, Reale G, Della Marca G, La Torre G, et al. Small-world characteristics of cortical connectivity changes in acute stroke.
Neurorehabil Neural Repair. (2017) 31:81-94. doi: 10.1177/154596831666 2525

49. Nielsen JA, Zielinski BA, Ferguson MA, Lainhart JE, Anderson JS. An evaluation of the left-brain vs. right-brain hypothesis with resting state functional connectivity magnetic resonance imaging. PLoS ONE. (2013) 8:e71275. doi: 10.1371/journal.pone.0071275

50. Kumar V, Mang S, Grodd W. Direct diffusion-based parcellation of the human thalamus. Brain Struct Funct. (2015) 220:161935. doi: 10.1007/s00429-014-0748-2

51. Bressler SL, Tang W, Sylvester CM, Shulman GL, Corbetta M. Topdown control of human visual cortex by frontal and parietal cortex in anticipatory visual spatial attention. J Neurosci. (2008) 28:1005661. doi: 10.1523/JNEUROSCI.1776-08.2008

52. Tunik E, Saleh S, Adamovich SV. Visuomotor discordance during visuallyguided hand movement in virtual reality modulates sensorimotor cortical activity in healthy and hemiparetic subjects. IEEE Trans Neural Syst Rehabil Eng. (2013) 21:198-207. doi: 10.1109/TNSRE.2013.2238250

53. Brewer BR, Klatzky R, Matsuoka Y. Visual feedback distortion in a robotic environment for hand rehabilitation. Brain Res Bull. (2008) 75:80413. doi: 10.1016/j.brainresbull.2008.01.006

54. Kang N, Cauraugh J. Bimanual force variability in chronic stroke: with and without visual information. Neurosci Lett. (2015) 587:415. doi: 10.1016/j.neulet.2014.12.028

55. Westerveld A, Schouten A, Veltink P, van der Kooij H. Control of thumb force using surface functional electrical stimulation and muscle load sharing. J Neuroeng Rehabil. (2013) 10:104. doi: 10.1186/1743-0003-10-104

56. Archer DB, Kang N, Misra G, Marble S, Patten C, Coombes SA. Visual feedback alters force control and functional activity in the visuomotor network after stroke. NeuroImage Clinical. (2018) 17:50517. doi: 10.1016/j.nicl.2017.11.012

57. Tal Z, Geva R, Amedi A. The origins of metamodality in visual object area LO: bodily topographical biases and increased funct ional connectivity to S1. Neuroimage. (2016) 127:363-75. doi: 10.1016/j.neuroimage.2015.11.058

58. Rehme AK, Grefkes C. Cerebral network disorders after stroke: evidence from imaging-based connectivity analyses of active and resting brain states in humans. J Physiol. (2013) 591:17-31. doi: 10.1113/jphysiol.2012.243469

59. Hu J, Du J, Xu Q, Yang F, Zeng F, Weng Y, et al. Dynamic network analysis reveals altered temporal variability in brain regions after stroke: a longitudinal resting-state fMRI study. Neural Plast. (2018) 2018:9394156. doi: 10.1155/2018/9394156

60. Li X, Liang Y, Chen Y, Zhang J, Wei D, Chen K, et al. Disrupted frontoparietal network mediates white matter structure dysfunction associated with cognitive decline in hypertension patients. J Neurosci. (2015) 35:1001524. doi: 10.1523/JNEUROSCI.5113-14.2015

61. Demuru M, van Duinkerken E, Fraschini M, Marrosu F, Snoek FJ, Barkhof $\mathrm{F}$, et al. Changes in MEG resting-state networks are related to cognitive decline in type 1 diabetes mellitus patients. NeuroImage Clin. (2014) 5:69-76. doi: 10.1016/j.nicl.2014. 06.001

Conflict of Interest: The authors declare that the research was conducted in the absence of any commercial or financial relationships that could be construed as a potential conflict of interest.

Publisher's Note: All claims expressed in this article are solely those of the authors and do not necessarily represent those of their affiliated organizations, or those of the publisher, the editors and the reviewers. Any product that may be evaluated in this article, or claim that may be made by its manufacturer, is not guaranteed or endorsed by the publisher.

Copyright (C) 2022 Huang, Lin, Huang, Cao, Jin, Wu, Fan, Tu, Huang and Jiang. This is an open-access article distributed under the terms of the Creative Commons Attribution License (CC BY). The use, distribution or reproduction in other forums is permitted, provided the original author(s) and the copyright owner(s) are credited and that the original publication in this journal is cited, in accordance with accepted academic practice. No use, distribution or reproduction is permitted which does not comply with these terms. 